\title{
Images - Computed tomography urographic appearance of traumatic rupture of renal cyst into the pyelocaliceal system
}

\author{
Elena Vetrano${ }^{1}$; Dario Giambelluca ${ }^{1}$; Massimo Midiri ${ }^{1}$; Marco Vella²; Giuseppe Salvaggio ${ }^{1}$ \\ ${ }^{1}$ Department of Radiological Sciences, University of Palermo, Palermo, Italy; ${ }^{2}$ Department of Urology, University of \\ Palermo, Palermo, Italy
}

Cite as: Can Urol Assoc J 2019 September 27; Epub ahead of print. http://dx.doi.org/10.5489/cuaj.5937

Published online September 27, 2019

\section{Introduction}

Rupture of renal cyst can occur with trauma or increased intracystic pressure, secondary to cyst hemorrhage or infection. ${ }^{1}$ Cystic rupture may drain into the pyelocaliceal system, the perirenal space, or the peritoneal cavity. ${ }^{1,2}$ Symptoms include flank pain and hematuria, or may be entirely absent. ${ }^{2,3,4}$ Hematuria is seen in up to $84 \%$ of patients with cyst rupture, likely resulting from drainage into an adjacent calyx. ${ }^{4}$ Ultrasound examination is generally the first diagnostic approach to the trauma patient; however, it has low sensitivity for ruptured renal cysts, since some cystic cavities contain infected debris or blood clot, so that they may appear as complex masses on the sonogram. ${ }^{5}$ Computed tomography (CT) urography is the imaging technique of choice to ensure an accurate diagnosis and an appropriate management of complicated renal cysts. Normally, cysts have density values of water, with an attenuation coefficient between 0 and 20 Hounsfield units (HU) on unenhanced CT images, and do not enhance after contrast medium administration. ${ }^{6}$ The cystic cavity is usually subcapsular and abuts directly against the renal pelvis or calix. ${ }^{7}$ It is essential to differentiate a ruptured renal cyst from pyelocaliceal diverticula, which are corticomedullary in location and have elongated, narrow necks. ${ }^{5}$ During delayed phase images, the cyst appears to have a smooth or lobular inner wall, depending on the degree of cystic distension with contrast material. Filling defects within the lumen of the cyst may be blood clots or infected debris. ${ }^{5,7}$

The published literature contains only sporadic reports of communication between a renal cyst and the pyelocaliceal system. ${ }^{7,8,9,10}$ We report a case of a 77 -year-old male, admitted to our emergency department complaining of post-traumatic hematuria after he got into a car crash a few hours before. Renal ultrasonography detected a centimetric hyperechoic image inside a cyst in the right kidney, suspected for a blood clot (Figure 1). Then, a CT urography examination was performed. The unenhanced CT images revealed, in the upper polar site of the right kidney, a cyst with a hyperattenuating focus inside, consistent with blood clot. This cyst, in the delayed excretory phases, showed progressive intraluminal contrast enhancement, with subtle communication with the medium calyceal group (Figure 2). The patient was treated with potassium chloride solution, antibiotic coverage, antihypertensive therapy and a bladder catheter was placed. At 6-day followup, he was asymptomatic on clinical examination and a new CT urography was performed, revealing the reduction in diameter of the renal cyst (Figure 3A). However, persistence of 
communication between pyelocaliceal system and renal cyst was reported. The patient was discharged in good general clinical conditions, with spontaneous urination and clear urine. At 3month follow-up, another CT urography was performed, and the previously detected ruptured renal cyst was no longer visible (Figure 3B).

\section{Conclusions}

Spontaneous or traumatic rupture of renal cysts, documented by radiological imaging, occurs infrequently. ${ }^{1}$ The pathogenesis of a cyst rupture is still unclear, except when it has occurred following trauma or when the cyst has become infected. ${ }^{5,7}$ Imaging plays a crucial role in the diagnosis, management, surveillance and choice of lesions that need treatment.,

Following cystic rupture, the outcome varies from rapid closure of the cyst communication (with or without collapse of the cyst cavity) to its persistence and the continuous filling of the cyst cavity during subsequent studies with intravenous contrast medium. ${ }^{7}$

In these patients, a conservative medical management must be the first option, since closure of the torn wall usually occurs within 3 days with cessation of bleeding, while it may not occur for weeks or months in other instances. ${ }^{1,5,7}$ Percutaneous renal puncture, percutaneous nephrostomy or the ureteral stenting should be reserved for special cases with refractory pain, urinary obstruction or poor evolution. ${ }^{1,5,7}$ 


\section{References}

1. Garcia-Segui A, Ortiz-Gorraiz M, Soler-López C, et al. Rupture of a simple renal cyst into the pyelocalyceal system induced by laparoscopic surgery of the prostate. Ann Surg Perioper Care 2016;1:1015

2. Nussbaum A, Hunter TB, Stables DP. Spontaneous cyst rupture on renal CT. AJR Am J Roentgenol 1984;142:751-2

3. Marques DT, Bezerra RO, de Brito Siqueira LT, et al. Spontaneous combined rupture of a pelvicalyceal cyst into the collector system and retroperitoneal space during the acquisition of computed tomography scan images: a case report. J Med Case Rep 2012;13:386

4. Lee J, Darcy M. Renal cysts and urinomas. Semin Intervent Radiol 2011;28:380-91

5. McLaughlin AP 3rd, Pfister RC. Spontaneous rupture of renal cysts into the pyelocaliceal system. J Urol 1975;113:2-7

6. Galia M, Albano D, Bruno A, et al. Imaging features of solid renal masses. Br J Radiol 2017;90:20170077

7. Papanicolaou N, Pfister RC, Yoder IC. Spontaneous and traumatic rupture of renal cysts: diagnosis and outcome. Radiology 1986;160:99-103

8. Reiss MD. Traumatic rupture of renal cortical cyst into the calyceal system. Am J Roentgenol Radium Ther Nucl Med 1967;101:696-9

9. Mayer DP, Baron RL, Pollack HM. Increase in CT attenuation values of parapelvic renal cysts after retrograde pyelography. AJR Am J Roentgenol 1982;139:991-3

10. Davis JM, McLaughlin AP. Spontaneous renal hemorrhage due to cyst rupture: CT findings. AJR Am J Roentgenol 1987;148:763-49. 


\section{Figures and Tables}

Fig. 1. Ultrasound image shows multiple cystic lesions (curved arrows) in the right kidney. Note a hyperechoic image (arrowhead) within the lumen of a renal cyst, consistent with a blood clot.

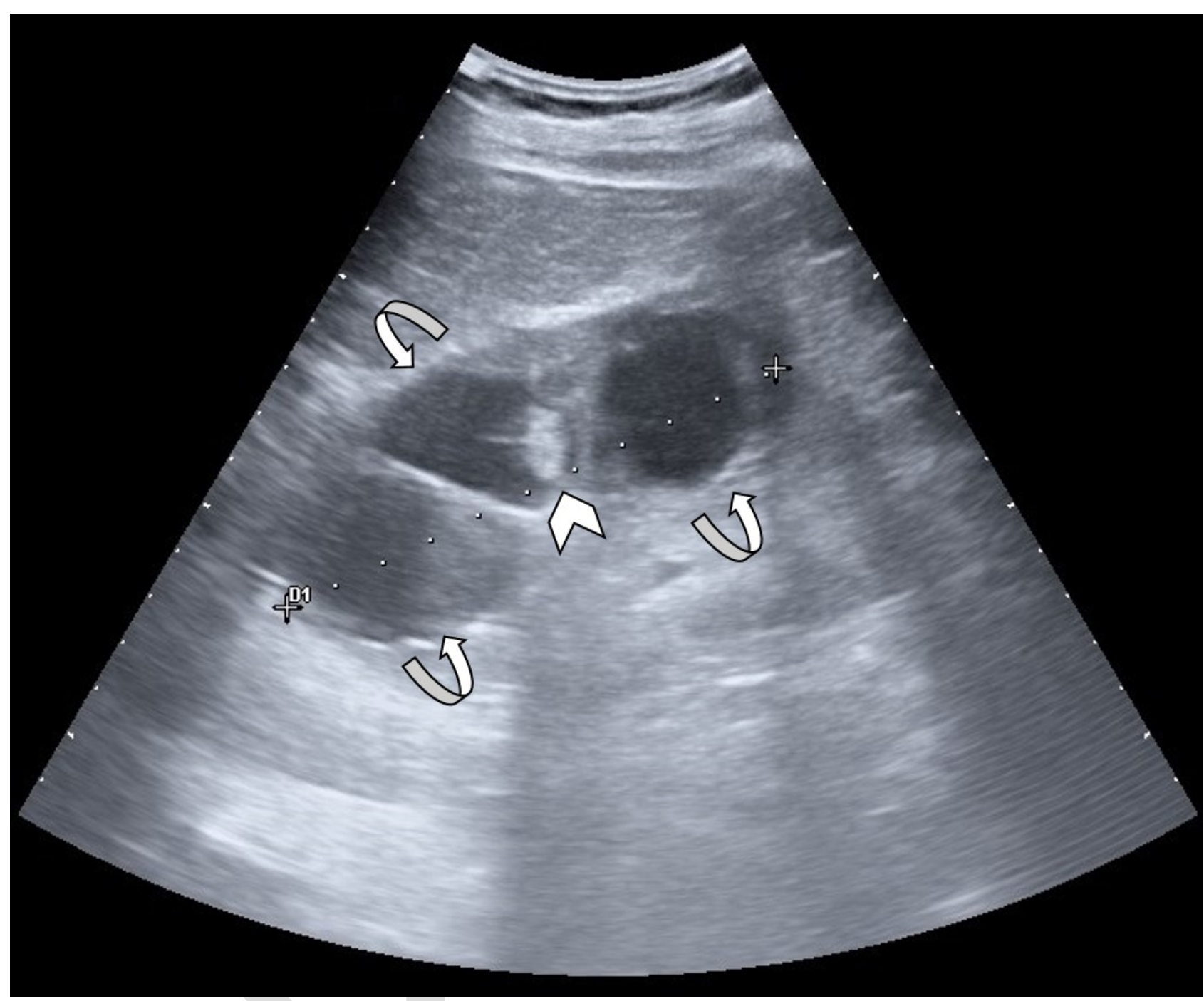


Fig 2. (A, B) Unenhanced computed tomography (CT) images show a cyst with blood clot inside (arrowhead) in the right kidney. Note a subcapsular hematoma in the anterior mesorenal cortex (curved arrow). (C) CT urography in portal phase shows the absence of contrast enhancement within the cyst. CT images in delayed excretory phase at $(\boldsymbol{D}) 5$ and $(\boldsymbol{E}) 10$ minutes show the progressive cyst filling (arrows). (F) Coronal reformatted CT image, with maximum intensity projection, depicts a subtle communication (arrowhead), with contrast medium spilling from the medium calyceal group to the cyst.

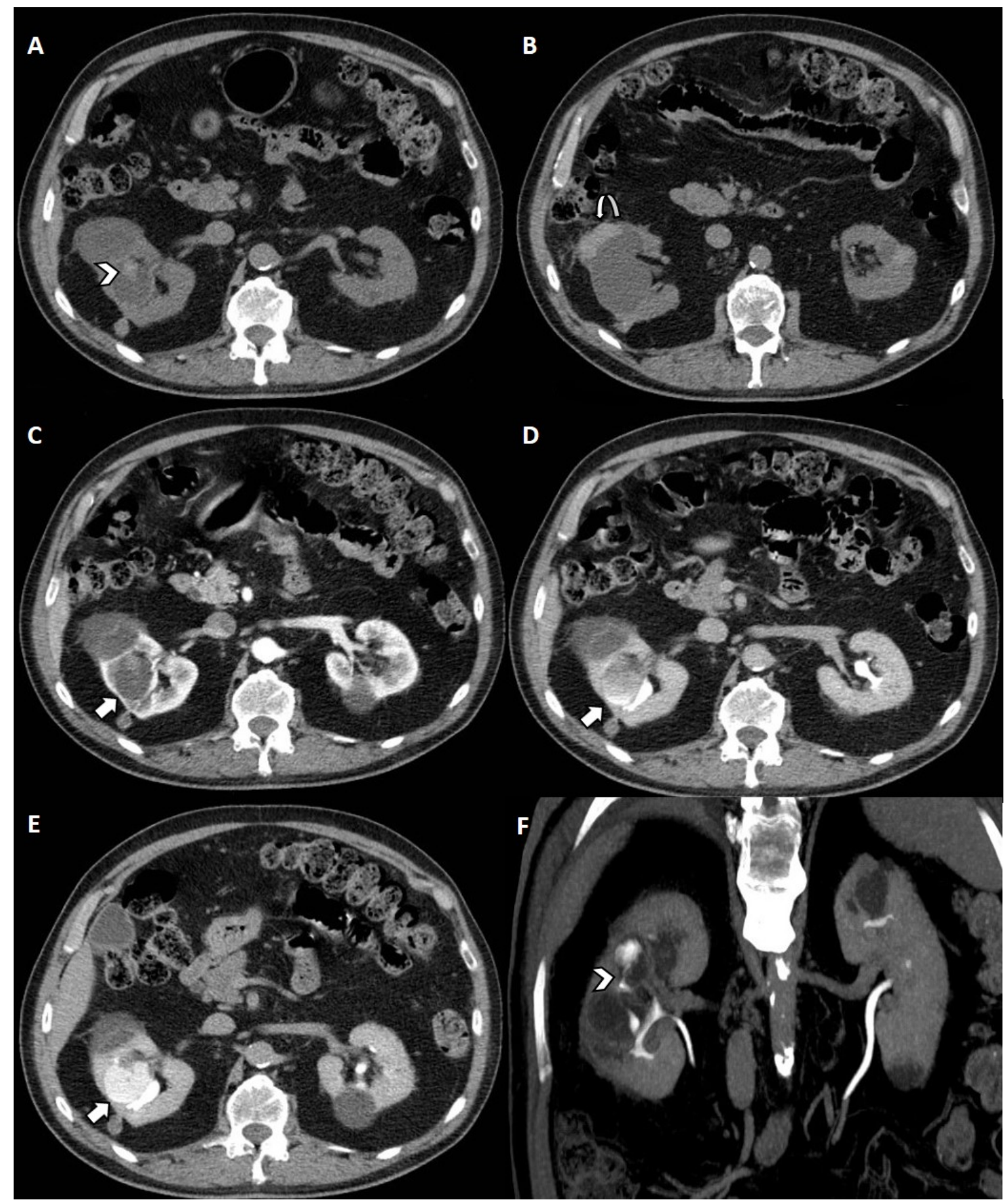


Fig. 1. (A) Six days' followup computed tomography (CT) urography in delayed excretory phase (5 minutes) shows the direct communication (arrowhead) between the medium calyceal group and the ruptured renal cyst. Note the backflow of contrast medium from the pyelocaliceal system into the ruptured cyst cavity, which has become smaller (from maximum diameter of $4 \mathrm{~cm}$ to $3.2 \mathrm{~cm}$ ). (B) CT urography in delayed excretory phase (5 minutes), obtained three months after the first examination, shows the disappearance of the cyst cavity (arrow), with no more imaging appearance of the pyelocaliceal communication.

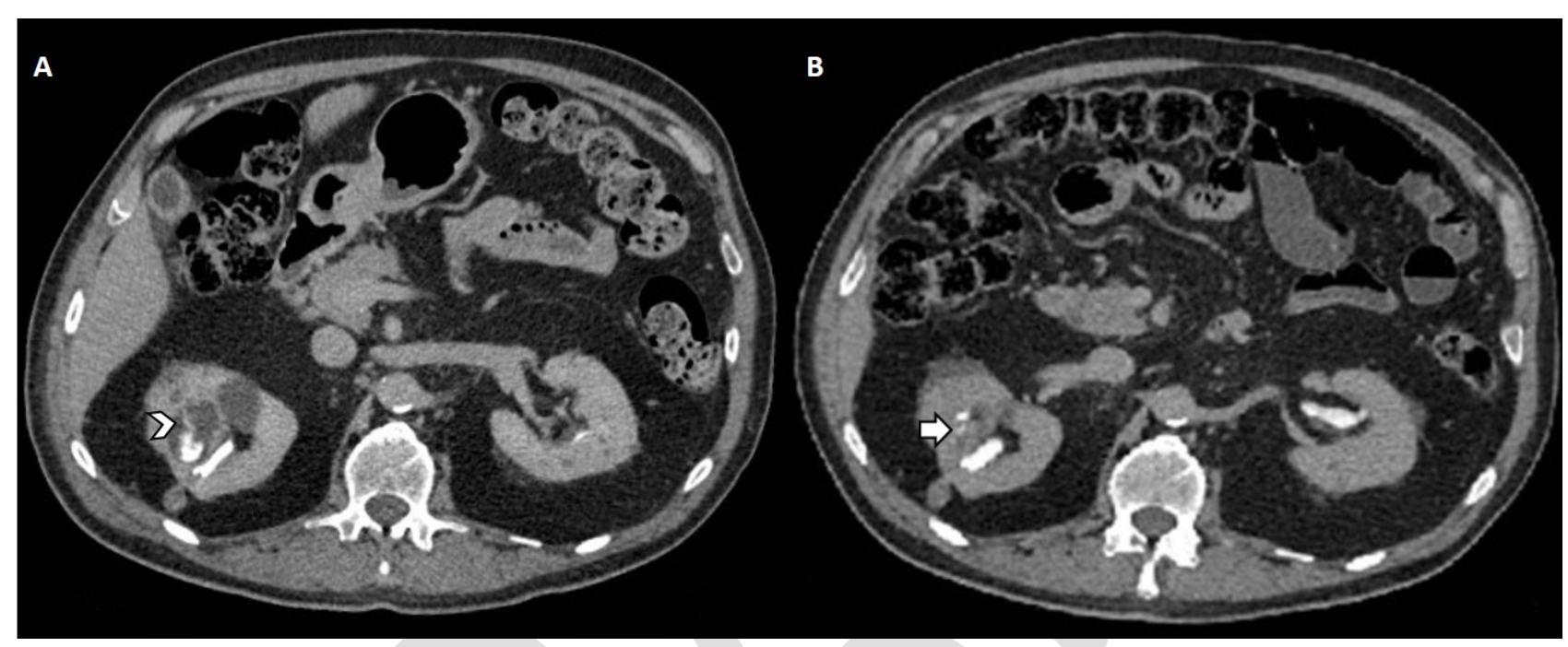

